Applied Mathematical Sciences, Vol. 7, 2013, no. 133, 6601 - 6609

HIKARI Ltd, www.m-hikari.com

http://dx.doi.org/10.12988/ams.2013.310559

\title{
Exponentially Small Expansions of the Confluent Hypergeometric Functions
}

\author{
R. B. Paris
}

School of Engineering, Computing and Applied Mathematics

University of Abertay Dundee, Dundee DD1 1HG, UK

Copyright (c) 2013 R. B. Paris. This is an open access article distributed under the Creative Commons Attribution License, which permits unrestricted use, distribution, and reproduction in any medium, provided the original work is properly cited.

\begin{abstract}
The asymptotic expansions of the confluent hypergeometric functions ${ }_{1} F_{1}(a ; b ; z)$ and $U(a, b, z)$ are examined as $|z| \rightarrow \infty$ on the Stokes lines $\arg z= \pm \pi$. Particular attention is given to the exponentially small contributions associated with these two functions. Numerical results demonstrating the accuracy of the expansions are given.
\end{abstract}

Mathematics Subject Classification: 30E15, 33C15, 34E05, 41A60

Keywords: Confluent hypergeometric functions, Stokes lines, asymptotic expansions, exponentially small expansions

\section{Introduction}

The confluent hypergeometric function ${ }_{1} F_{1}(a ; b ; z)$ (or first Kummer function also denoted by $M(a, b, z))$ is defined for complex parameters $a$ and $b$ by

$$
{ }_{1} F_{1}(a ; b ; z)=\sum_{r=0}^{\infty} \frac{(a)_{r} z^{r}}{(b)_{r} r !}=1+\frac{a z}{b 1 !}+\frac{a(a+1) z^{2}}{b(b+1) 2 !}+\ldots,
$$

provided $b \neq 0,-1,-2, \ldots$, where $(a)_{r}=a(a+1) \ldots(a+r-1)=\Gamma(a+r) / \Gamma(a)$ is Pochhammer's symbol for the rising factorial. The series converges absolutely for all finite $z$ and reduces to a polynomial in $z$ when $a=0,-1,-2, \ldots$. 
We exclude this last case from our asymptotic considerations. The function ${ }_{1} F_{1}(a ; b ; z)$ is entire in $z$ and is consequently completely described in $-\pi<\arg z \leq \pi$.

The behaviour of ${ }_{1} F_{1}(a ; b ; z)$ for large $z$ and fixed parameters is exponentially large in $\operatorname{Re}(z)>0$ and algebraic in character in $\operatorname{Re}(z)<0$. The well-known asymptotic expansion of ${ }_{1} F_{1}(a ; b ; z)$ for $|z| \rightarrow \infty$ is given by $[4$, p. 328]

$$
\frac{\Gamma(a)}{\Gamma(b)}{ }_{1} F_{1}(a ; b ; z) \sim E(z)+H\left(z e^{\mp \pi i}\right) \quad\left(-\frac{1}{2} \pi+\epsilon \leq \pm \arg z \leq \frac{3}{2} \pi-\epsilon\right),
$$

where throughout $\epsilon>0$ denotes an arbitrarily small quantity. Here the formal exponential and algebraic asymptotic series $E(z)$ and $H(z)$ are defined by

$$
E(z):=z^{a-b} e^{z} \sum_{j=0}^{\infty} A_{j} z^{-j}, \quad H(z):=z^{-a} \frac{\Gamma(a)}{\Gamma(b-a)} \sum_{k=0}^{\infty} \frac{(a)_{k}(1+a-b)_{k}}{k ! z^{k}}
$$

where the coefficients $A_{j}$ are given by

$$
A_{j}=\frac{(1-a)_{j}(b-a)_{j}}{j !} \quad(j \geq 0) .
$$

The exponential expansion $E(z)$ is dominant as $|z| \rightarrow \infty$ in $\operatorname{Re}(z)>$ 0 and becomes oscillatory on the anti-Stokes lines $\arg z= \pm \frac{1}{2} \pi$, where it is of comparable magnitude to the algebraic expansion. $\operatorname{In} \operatorname{Re}(z)<0$, the exponential expansion is subdominant with the behaviour of ${ }_{1} F_{1}(a ; b ; z)$ then controlled by $H\left(z e^{\mp \pi i}\right)$. The negative real axis $\arg z= \pm \pi$ is a Stokes line where $E(z)$ is maximally subdominant. When $H\left(z e^{\mp \pi i}\right)$ is optimally truncated at, or near, its least term, the exponential expansion undergoes a smooth but rapid transition in the neighbourhood of $\arg z= \pm \pi$; see [4, p. 67] and [6, Chapter 6]. It is clear, however, that (1.1) cannot account correctly for the exponentially small expansion on $\arg z=\pi$, since it predicts the exponentially small behaviour $\left(|z| e^{\pi i}\right)^{a-b} e^{-|z|}$ as $|z| \rightarrow \infty$. When $a-b$ is non-integer with $a$ and $b$ real, this is a complex-valued contribution whereas ${ }_{1} F_{1}(a ; b ;-|z|)$ is real.

The interest in exponentially precise asymptotics during the past two decades has shown that retention of exponentially small terms, previously neglected in asymptotics, is essential for a high-precision description. An early example that illustrated the advantage of retaining exponentially small terms in the asymptotic expansion of a certain integral was given in Olver's wellknown book [1, p. 76]. Although such terms are negligible in the Poincaré sense, their inclusion can significantly improve the numerical accuracy.

Our aim in this note is to obtain the exponentially small expansion associated with ${ }_{1} F_{1}(a ; b ;-x)$, and also that of the second Kummer function 
$U\left(a, b, x e^{ \pm \pi i}\right)$, as $x \rightarrow+\infty$ when the dominant algebraic expansion is optimally truncated.

\section{The expansion for ${ }_{1} F_{1}(a ; b ;-x)$ as $x \rightarrow+\infty$}

The second Kummer function $U(a, b, z)$ is defined as a linear combination of two ${ }_{1} F_{1}$ functions $[4$, p. 325] and has, in general, a branch point at $z=0$ with the $z$-plane cut along $(-\infty, 0]$. This function possesses the large- $|z|$ expansion

$$
U(a, b, z) \sim z^{-a} \sum_{k=0}^{\infty} \frac{(-)^{k}(a)_{k}(1+a-b)_{k}}{k ! z^{k}} \quad\left(|\arg z| \leq \frac{3}{2} \pi-\epsilon\right),
$$

which is algebraic in character in the stated sector. However, on the Stokes lines $\arg z= \pm \pi$, an exponentially small expansion switches on, so that in the sectors $\pi \leq|\arg z| \leq \frac{3}{2} \pi-\epsilon$ we have a compound expansion with a subdominant exponential contribution. This latter contribution becomes dominant beyond $|\arg z|=\frac{3}{2} \pi$. In the cases $a, 1+a-b=-n, n=0,1,2, \ldots, z^{a} U(a, b, z)$ reduces to a polynomial in $z^{-1}$ of degree $n$; see [4, p. 322].

In the following we let $M, N, m$ denote positive integers and define the parameters

$$
\vartheta:=a-b, \quad \nu \equiv \nu(m):=a+m+\vartheta .
$$

The exponentially improved expansion of $U(a, b, z)$ is given by [4, p. 329]

$$
\begin{aligned}
U(a, b, z)=z^{-a} \sum_{k=0}^{m-1} & \frac{(-)^{k}(a)_{k}(1+a-b)_{k}}{k ! z^{k}} \\
& +\frac{2 \pi i e^{-\pi i(a+\vartheta)}}{\Gamma(a) \Gamma(1+a-b)} z^{\vartheta} e^{z}\left\{\sum_{j=0}^{M-1} A_{j} z^{-j} T_{\nu-j}(z)+R_{M, m}(z)\right\},
\end{aligned}
$$

where the $A_{j}$ are defined in (1.3), $T_{\nu}(z)$ denotes the so-called terminant function defined as a multiple of the incomplete gamma function $\Gamma(a, z)$ by

$$
T_{\nu}(z):=\frac{e^{\pi i \nu} \Gamma(\nu)}{2 \pi i} \Gamma(1-\nu, z)
$$

In (2.2) $m$ is an arbitrary positive integer but will be chosen to be the optimal truncation index $m_{o}$ of the algebraic expansion corresponding to truncation at, or near, the least term in magnitude. This is easily verified to be

$$
m_{o} \simeq|z|-\operatorname{Re}(a+\vartheta)
$$

so that $m_{o} \rightarrow \infty$ as $|z| \rightarrow+\infty$. When $m-|z|$ is bounded, the remainder term in (2.2) satisfies $R_{M, m}(z)=O\left(e^{-|z|-z} z^{-M}\right)$ as $|z| \rightarrow \infty$ in the sector 
$|\arg z| \leq \pi$. The expansion (2.2) was first obtained by Olver [2, 3] who based his analysis on a Laplace integral representation for $U(a, b, z)$. A different derivation using a Mellin-Barnes integral representation was given in [5]; see also [6, p. 241 et seq.].

\subsection{The expansion when $\vartheta$ is non-integer}

The analogous expansion for ${ }_{1} F_{1}(a ; b ;-x)$ for $x \rightarrow+\infty$ can be obtained by making use of the identity $[4$, p. 323]

$$
{ }_{1} F_{1}(a ; b ; z)=\frac{\Gamma(1+a-b) \Gamma(b)}{2 \pi i}\left\{e^{\pi i b} U(a, b, z)-e^{-\pi i b} U\left(a, b, z e^{-2 \pi i}\right)\right\} .
$$

Then, with $z=x e^{\pi i}, x>0$, substitution of the expansion (2.2) into the above identity yields

$$
\begin{aligned}
& \frac{\Gamma(a)}{\Gamma(b)}{ }_{1} F_{1}(a ; b ;-x)=\frac{\Gamma(a) \Gamma(1+a-b)}{2 \pi i}\left\{e^{\pi i b} U\left(a, b, x e^{\pi i}\right)-e^{-\pi i b} U\left(a, b, x e^{-\pi i}\right)\right\} \\
& =\frac{x^{-a} \Gamma(a)}{\Gamma(b-a)} \sum_{k=0}^{m_{o}-1} \frac{(a)_{k}(1+a-b)_{k}}{k ! x^{k}}+x^{\vartheta} e^{-x}\left\{\sum_{j=0}^{M-1}(-)^{j} A_{j} x^{-j} G_{j}(x)+O\left(x^{-M}\right)\right\}
\end{aligned}
$$

where

$$
G_{j}(x):=e^{-\pi i \vartheta}\left\{T_{\nu-j}\left(x e^{\pi i}\right)-e^{-2 \pi i a} T_{\nu-j}\left(x e^{-\pi i}\right)\right\} .
$$

Since the truncation index $m$ is chosen to be optimal, the index $\nu$ appearing in (2.1) satisfies $\nu \sim x$ as $x \rightarrow+\infty$. The asymptotic expansion of $T_{\nu}(z)$ for large $|\nu|$ and $|z|$, when $|\nu| \sim|z|$, has been discussed in detail by Olver in [2]. By expressing $T_{\nu}(z)$ in terms of a Laplace integral, which is associated with a saddle point and a simple pole becoming coincident on $\arg z=\pi$, Olver $[2$, $\S 5]$ established that

$$
T_{\nu-j}\left(x e^{\pi i}\right)=\frac{1}{2}-\frac{i}{\sqrt{2 \pi x}}\left\{\sum_{k=0}^{N-1}\left(\frac{1}{2}\right)_{k} g_{2 k}(j)\left(\frac{1}{2} x\right)^{-k}+O\left(x^{-N}\right)\right\} \quad(x \rightarrow+\infty),
$$

where the coefficients $g_{k}(j)$ result from the expansion

$$
\frac{\tau^{\gamma_{j}-1}}{1-\tau} \frac{d \tau}{d w}=-\frac{1}{w}+\sum_{k=0}^{\infty} g_{k}(j) w^{k}, \quad \frac{1}{2} w^{2}=\tau-\log \tau-1
$$

The branch of $w(\tau)$ is chosen such that $w \sim \tau-1$ as $\tau \rightarrow 1$ and the parameter $\gamma_{j}$ is specified by

$$
\gamma_{j}=\nu-x-j \quad(0 \leq j \leq N-1)
$$


with $\left|\gamma_{j}\right|$ bounded. Upon reversion of the $w-\tau$ mapping to yield

$$
\tau=1+w+\frac{1}{3} w^{2}+\frac{1}{36} w^{3}-\frac{1}{270} w^{4}+\frac{1}{4320} w^{5}+\cdots,
$$

it is found with the help of Mathematica that the first five even-order coefficients $g_{2 k}(j) \equiv 6^{-2 k} \hat{g}_{2 k}(j)$ are

$$
\begin{aligned}
& \hat{g}_{0}(j)= \frac{2}{3}-\gamma_{j}, \quad \hat{g}_{2}(j)=\frac{1}{15}\left(46-225 \gamma_{j}+270 \gamma_{j}^{2}-90 \gamma_{j}^{3}\right), \\
& \hat{g}_{4}(j)= \frac{1}{70}\left(230-3969 \gamma_{j}+11340 \gamma_{j}^{2}-11760 \gamma_{j}^{3}+5040 \gamma_{j}^{4}-756 \gamma_{j}^{5}\right), \\
& \hat{g}_{6}(j)=\frac{1}{350}\left(-3226-17781 \gamma_{j}+183330 \gamma_{j}^{2}-397530 \gamma_{j}^{3}+370440 \gamma_{j}^{4}-170100 \gamma_{j}^{5}\right.\left.+37800 \gamma_{j}^{6}-3240 \gamma_{j}^{7}\right), \\
& \hat{g}_{8}(j)=\frac{1}{231000}\left(-4032746+43924815 \gamma_{j}+88280280 \gamma_{j}^{2}-743046480 \gamma_{j}^{3}\right. \\
& \quad+1353607200 \gamma_{j}^{4}-1160830440 \gamma_{j}^{5}+541870560 \gamma_{j}^{6}-141134400 \gamma_{j}^{7} \\
&\left.+19245600 \gamma_{j}^{8}-1069200 \gamma_{j}^{9}\right) .
\end{aligned}
$$

If we now make use of the connection formula ${ }^{1}$ [6, p. 260]

$$
T_{\nu}\left(x e^{-\pi i}\right)=e^{2 \pi i \nu}\left\{T_{\nu}\left(x e^{\pi i}\right)-1\right\}
$$

the quantities $G_{j}(x)$ involving the terminant functions in (2.5) become

$$
\begin{aligned}
G_{j}(x) & =e^{-\pi i \vartheta} T_{\nu-j}\left(x e^{\pi i}\right)-e^{\pi i \vartheta}\left\{T_{\nu-j}\left(x e^{\pi i}\right)-1\right\} \\
& =\cos \pi \vartheta-\frac{2 \sin \pi \vartheta}{\sqrt{2 \pi x}}\left\{\sum_{k=0}^{N-1}\left(\frac{1}{2}\right)_{k} g_{2 k}(j)\left(\frac{1}{2} x\right)^{-k}+O\left(x^{-N}\right)\right\}
\end{aligned}
$$

upon use of (2.6). Substitution of (2.8) into (2.4) (where for convenience we put $M=N)$ and introduction of the coefficients $B_{j}$ defined by

$$
B_{j}=\sum_{k=0}^{j}(-2)^{k}\left(\frac{1}{2}\right)_{k} A_{j-k} g_{2 k}(j-k)
$$

then finally yields the exponentially small expansion $\mathcal{E}(x)$ in the form

$$
\mathcal{E}(x):=x^{\vartheta} e^{-x}\left\{\cos \pi \vartheta \sum_{j=0}^{M-1}(-)^{j} A_{j} x^{-j}-\frac{2 \sin \pi \vartheta}{\sqrt{2 \pi x}} \sum_{j=0}^{M-1}(-)^{j} B_{j} x^{-j}+O\left(x^{-M}\right)\right\}
$$

as $x \rightarrow+\infty$.

From (2.4) and (2.10), the expansion of ${ }_{1} F_{1}(a ; b ;-x)$ is then given by the following theorem.

\footnotetext{
${ }^{1}$ This easily follows from the corresponding connection formula for $\Gamma(a, z)$ in $[4,(8.2 .10)]$.
} 
Theorem 1 When $\vartheta=a-b$ is non-integer we have the expansion

$$
\begin{aligned}
& \frac{\Gamma(a)}{\Gamma(b)}{ }_{1} F_{1}(a ; b ;-x)-\frac{x^{-a} \Gamma(a)}{\Gamma(b-a)} \sum_{k=0}^{m_{o}-1} \frac{(a)_{k}(1+a-b)_{k}}{k ! x^{k}} \\
& \quad=x^{\vartheta} e^{-x}\left\{\cos \pi \vartheta \sum_{j=0}^{M-1}(-)^{j} A_{j} x^{-j}-\frac{2 \sin \pi \vartheta}{\sqrt{2 \pi x}} \sum_{j=0}^{M-1}(-)^{j} B_{j} x^{-j}+O\left(x^{-M}\right)\right\}
\end{aligned}
$$

as $x \rightarrow+\infty$, where $m_{o}$ is the optimal truncation index of the algebraic expansion satisfying $m_{o} \sim x, M$ is a positive integer and the coefficients $A_{j}$ and $B_{j}$ are defined in (1.3) and (2.9) respectively.

\subsection{The expansion when $\vartheta$ is an integer}

When $\vartheta=n, n=0,1,2, \ldots$, it is seen from (1.2) that the algebraic expansion $H(z) \equiv 0$ and the coefficients $A_{j}$ in the exponential expansion vanish for $j>n$. From Kummer's transformation

$$
{ }_{1} F_{1}(a ; b ; z)=e^{z}{ }_{1} F_{1}(b-a ; b ;-z),
$$

the hypergeometric function on the right-hand side is a polynomial in $z$ of degree $n$. Upon some straightforward rearrangement we find the finite exponential series given by

$$
\frac{\Gamma(a)}{\Gamma(b)}{ }_{1} F_{1}(a ; b ; z)=z^{\vartheta} e^{z} \sum_{j=0}^{n}(-)^{j} A_{j}^{\prime} z^{-j}, \quad A_{j}^{\prime}=(1-a)_{j}\left(\begin{array}{l}
n \\
j
\end{array}\right)
$$

valid for all finite $z$, where the coefficients $A_{j}^{\prime}$ follow from the $A_{j}$ in (1.3).

When $\vartheta=-n$, the algebraic expansion consists of $n$ terms and so cannot be optimally truncated; the parameter $\nu$ in (2.1) (with $m=n$ ) is therefore finite in this limit. From the equation immediately above (2.8) it is seen that $G_{j}(x)=e^{\pi i \vartheta}=(-)^{n}$ in this case. Consequently, we then obtain the result given in the following theorem.

Theorem 2 Let $n$ and $M$ denote positive integers. For values of the parameter $\vartheta=a-b=-n$ we have the expansion

$$
\begin{aligned}
& \frac{\Gamma(a)}{\Gamma(b)}{ }_{1} F_{1}(a ; b ;-x)-\frac{x^{-a} \Gamma(a)}{\Gamma(b-a)} \sum_{k=0}^{n-1} \frac{(a)_{k}(1+a-b)_{k}}{k ! x^{k}} \\
& =x^{\vartheta} e^{-x}\left\{\cos \pi \vartheta \sum_{j=0}^{M-1}(-)^{j} A_{j} x^{-j}+O\left(x^{-M}\right)\right\}
\end{aligned}
$$

as $x \rightarrow+\infty$, where the coefficients $A_{j}$ are defined in (1.3); compare (1.1). 
It is seen that in the case $\vartheta=-n$ the exponentially small expansion of ${ }_{1} F_{1}(a ; b ;-x)$ is still given by $(2.10)$ since the second series involving the coefficients $B_{j}$ vanishes.

\section{Numerical examples and concluding remarks}

In this section we present some numerical examples to demonstrate the accuracy of the results obtained in Section 2. To demonstrate the validity of the expansions in $(2.11),(2.13)$ and (3.2) below we show in Table 1 the absolute relative errors ${ }^{2}$ in the computation of the exponentially small expansions when $x=15$ compared to their respective left-hand sides (with the algebraic expansion optimally truncated) for different summation index $M$. It was verified numerically that with this value of $x$ the range $M \leq 5$ corresponded to sub-optimal truncation of the exponentially small series.

Table 1: Values of the absolute relative error in the computation of the exponentially small expansions in (2.11), (2.13) and (3.2) when $x=15$ (with $m_{o}=15$ ) for different summation index $M$.

\begin{tabular}{|c|c|c|c|}
\hline \multirow[b]{2}{*}{$M$} & \multicolumn{2}{|c|}{${ }_{1} F_{1}(a ; b ;-x)$} & \multirow{2}{*}{$\begin{array}{c}U\left(a, b, x e^{\pi i}\right) \\
a=0.75, b=0.50\end{array}$} \\
\hline & $a=0.75, b=0.50$ & $a=0.50, b=2.50$ & \\
\hline 1 & $3.247(-3)$ & $6.203(-2)$ & $4.060(-3)$ \\
\hline 2 & $8.182(-5)$ & $8.773(-3)$ & $1.238(-4)$ \\
\hline 3 & $5.999(-6)$ & $1.848(-3)$ & $1.053(-5)$ \\
\hline 4 & $7.486(-7)$ & $5.125(-4)$ & $1.528(-6)$ \\
\hline 5 & $1.333(-7)$ & $1.759(-4)$ & $3.168(-7)$ \\
\hline
\end{tabular}

In Table 2 we show the values of the quantity $\mathcal{F}(x)$ defined by

$$
\mathcal{F}(x):=\frac{\Gamma(a)}{\Gamma(b)}{ }_{1} F_{1}(a ; b ;-x)-\frac{x^{-a} \Gamma(a)}{\Gamma(b-a)} \sum_{k=0}^{m_{o}-1} \frac{(a)_{k}(1+a-b)_{k}}{k ! x^{k}}
$$

for different values of $x$ and the corresponding exponentially small expansion $\mathcal{E}(x)$ in $(2.10)$ computed with the truncation index $M=5$. In each case the optimal truncation index $m_{o}$ of the algebraic expansion in $\mathcal{F}(x)$ was determined by inspection. In the case with $\vartheta=\frac{1}{2}$ we remark that the exponential series involving the coefficients $A_{j}$ makes no contribution to $\mathcal{E}(x)$. It can be seen that the computed values of $\mathcal{F}(x)$ agree well with the corresponding exponentially small expansions.

\footnotetext{
${ }^{2}$ In Tables 1 and 2 we write the values as $x(y)$ instead of $x \times 10^{y}$.
} 
Table 2: Values of $\mathcal{F}(x)$ and $\mathcal{E}(x)$ in (3.1) and (2.10) using an optimal truncation $m_{o} \sim x$ of the algebraic expansion and index $M=5$ for different values of $x$ and parameters $a, b$.

\begin{tabular}{r|cc|cc}
\hline & \multicolumn{2}{|c|}{$a=0.75, b=0.50(\vartheta=0.25)$} & $a=1.25, b=0.75$ & $(\vartheta=0.50)$ \\
$x$ & $\mathcal{F}(x)$ & $\mathcal{E}(x)$ & $\mathcal{F}(x)$ & $\mathcal{E}(x)$ \\
\hline 5 & $+8.035131(-3)$ & $+8.034497(-3)$ & $+5.853208(-4)$ & $+5.851079(-4)$ \\
10 & $+6.217278(-5)$ & $+6.217274(-5)$ & $+3.486634(-6)$ & $+3.486578(-6)$ \\
15 & $+4.564015(-7)$ & $+4.564014(-7)$ & $+2.245063(-8)$ & $+2.245057(-8)$ \\
20 & $+3.273714(-9)$ & $+3.273714(-9)$ & $+1.477388(-10)$ & $+1.477387(-10)$ \\
25 & $+2.317483(-11)$ & $+2.317483(-11)$ & $+9.811267(-13)$ & $+9.811265(-13)$ \\
\hline & $a=0.50, b=1.50(\vartheta=-1)$ & $a=0.50, b=0.75$ & $(\vartheta=-0.25)$ \\
$x$ & $\mathcal{F}(x)$ & $\mathcal{E}(x)$ & $\mathcal{F}(x)$ & $\mathcal{E}(x)$ \\
\hline 5 & $-1.240840(-3)$ & $-1.247194(-3)$ & $+3.559193(-3)$ & $+3.561678(-3)$ \\
10 & $-4.340627(-6)$ & $-4.341510(-6)$ & $+1.967339(-5)$ & $+1.967388(-5)$ \\
15 & $-1.977241(-8)$ & $-1.977300(-8)$ & $+1.182451(-7)$ & $+1.182455(-7)$ \\
20 & $-1.006538(-10)$ & $-1.006546(-10)$ & $+7.353202(-10)$ & $+7.353209(-10)$ \\
25 & $-5.450153(-13)$ & $-5.450167(-13)$ & $+4.658066(-12)$ & $+4.658067(-12)$ \\
\hline
\end{tabular}

We make three remarks on Theorems 1 and 2. First, the leading behaviour of the exponentially small expansion on the Stokes line is $\mathrm{O}\left(x^{\vartheta} e^{-x}\right)$ for $\vartheta$ not equal to half-integer values, but is $\mathrm{O}\left(x^{\vartheta-\frac{1}{2}} e^{-x}\right)$ when $\vartheta= \pm \frac{1}{2}, \pm \frac{3}{2}, \ldots$ Secondly, the so-called Stokes multiplier (given by the quantity in curly braces in (2.11) and (2.13)) is equal to $\cos \pi \vartheta$ to leading order. And thirdly, when $\vartheta=-n$, we see from (2.13) that the exponential series can also be written in the form

$$
\frac{1}{2}\left\{E\left(x e^{\pi i}\right)+E\left(x e^{-\pi i}\right)\right\},
$$

where $E(z)$ is defined in (1.2). When $\vartheta$ is non-integer, however, there is an additional contribution from the series involving the coefficients $B_{j}$.

The expansion for $U\left(a, b, x e^{ \pm \pi i}\right)$ follows from (2.2) and (2.6), with $m$ chosen to be the optimal truncation index $m_{o} \sim x$ according to (2.3), as

$$
\begin{aligned}
& U\left(a, b, x e^{ \pm \pi i}\right)-\left(x e^{ \pm \pi i}\right)^{-a} \sum_{k=0}^{m_{o}-1} \frac{(a)_{k}(1+a-b)_{k}}{k ! x^{k}} \\
& = \pm \frac{2 \pi i e^{\mp \pi i a} x^{\vartheta} e^{-x}}{\Gamma(a) \Gamma(1+a-b)}\left\{\frac{1}{2} \sum_{j=0}^{M-1}(-)^{j} A_{j} x^{-j} \mp \frac{i}{\sqrt{2 \pi x}} \sum_{j=0}^{M-1}(-)^{j} B_{j} x^{-j}+O\left(x^{-M}\right)\right\}
\end{aligned}
$$


as $x \rightarrow+\infty$, provided $a, 1+a-b \neq 0,-1,-2, \ldots$ The above result confirms the familiar fact that the value of the Stokes multiplier of $U(a, b, z)$ (given by the expression in curly braces in (3.2)) on $\arg z= \pm \pi$ is $\frac{1}{2}$ to leading order.

Finally, we remark that the exponentially small expansions associated with the Whittaker functions $M_{\kappa, \mu}(z)$ and $W_{\kappa, \mu}(z)$ defined by [4, p. 334]

$$
\begin{gathered}
M_{\kappa, \mu}(z)=z^{\frac{1}{2}+\mu} e^{-\frac{1}{2} z}{ }_{1} F_{1}\left(\frac{1}{2}+\mu-\kappa ; 1+2 \mu ; z\right) \quad(2 \mu \neq-1,-2, \ldots) \\
W_{\kappa, \mu}(z)=z^{\frac{1}{2}+\mu} e^{-\frac{1}{2} z} U\left(\frac{1}{2}+\mu-\kappa, 1+2 \mu, z\right)
\end{gathered}
$$

on the negative real $z$-axis can be immediately obtained from $(2.11),(2.13)$ and (3.2).

\section{References}

[1] F. W. J. Olver, Asymptotics and Special Functions, Academic Press, New York, 1974. Reprinted in A. K. Peters, Massachussets, 1997.

[2] F. W. J. Olver, Uniform, exponentially improved, asymptotic expansions for the generalized exponential integral, SIAM J. Math. Anal. 22 (1991) 1460-1474.

[3] F. W. J. Olver, Uniform, exponentially improved, asymptotic expansions for the confluent hypergeometric function and other integral transforms, SIAM J. Math. Anal. 22 (1991) 1475-1486.

[4] F. W. J. Olver, D. W. Lozier, R. F. Boisvert and C. W. Clark (eds.), NIST Handbook of Mathematical Functions, Cambridge University Press, Cambridge, 2010.

[5] R. B. Paris, Smoothing of the Stokes phenomenon using Mellin-Barnes integrals, J. Comput. Appl. Math. 41 (1992) 117-133.

[6] R. B. Paris and D. Kaminski, Asymptotics and Mellin-Barnes Integrals, Cambridge University Press, Cambridge, 2001.

Received: October 10, 2013 